\title{
Robust Estimation for ARCH Models
}

\author{
Beatriz Vaz de Melo Mendes* \\ Antonio Marcos Duarte Júnior**
}

\begin{abstract}
This article introduces the class of the constrained M-estimators for ARCH models. The new estimators are defined based on the minimization of a bounded function of the squared residuals standardized by a robust scale. Their robustness and efficiency properties are derived. Using Monte Carlo experiments, it is shown that under small percentages of contaminations the robust estimates are still able to capture the dynamics of the process. The robust procedure is used to estimate the volatility of four Brazilian financial series.
\end{abstract}

\section{Resumo}

Este artigo apresenta uma classe de estimadores robustos para modelos ARCH. Estes novos estimadores são definidos baseados na minimização de uma função limitada dos resíduos quadrados padronizados por um parâmetro de escala. As características de robustez e eficiência são apresentadas. Com o uso de simulação Monte Carlo, mostramos que para poucas contaminações os estimadores robustos são capazes de capturar a dinâmica inerente ao processo. Esta nova proposta robusta é usada também para a análise de quatros séries obtidas do mercado financeiro brasileiro.

Key Words: ARCH models; volatility; robust estimation; M-estimation; constrained M-estimation.

JEL Code: C13, C15 and C52.

${ }^{*}$ UFRJ. E-mail: bmendesdme.ufrj.br.

** UFRJ.

\begin{tabular}{llll}
\hline R. de Econometria & Rio de Janeiro & v. $19, \mathrm{n}^{\mathrm{O}}$ 1, pp. 139-180 & Maio 1999 \\
\hline
\end{tabular} 


\section{Introduction}

The Autoregressive Conditional Heteroscedastic (ARCH) processes (Engle, 1982) and their subsequent generalizations (Bollerslev, 1986, Engle et al., 1987, etc.) are very useful when modeling heteroscedasticity in financial data.

Empirical evidence that the unconditional distribution of the returns of assets/indexes may have third and fourth moments not compatible with those implied by a conditional Gaussian specification was found in many applications. In those cases, the concern about deviations from normality was often translated into the use of a conditional t-student. Examples where high skewness and kurtosis were modeled using a conditional t-student distribution include Bollerslev (1987), Badrinath and Chatergee (1988), Gallant et al. (1989), Engle and González-Rivera (1991) and Nelson (1991).

Financial data typically possess heavy tails, present volatility clusters and occasionally show unexpected extreme data points. These events may be explained by GARCH-type models such as the EGARCH (Nelson, 1991) or the TGARCH (Rabemananjara and Zakoian, 1993, and Zakoian, 1991). Figure 1 gives an example of a financial series possessing these characteristics. This figure shows the daily returns of one of the series used in section 6 , the Brazilian Brady bond C-bond.

In Finance, a great importance is certainly given to extreme outlying points. However, these points may distort the maximum likelihood estimates (Huber, 1981). For example, Engle and GonzálezRivera (1991) reported the loss of efficiency of the quasi-maximum likelihood estimator (QMLE) of GARCH models. Evidence of bias on the QMLE in small samples was studied in Engle et al. (1985), Bollerslev and Wooldridge (1992) and Lumsdaine (1995). Mendes (1996) quantified the lack of robustness of the QMLE for ARCH 
models. Deb (1996) examined the finite sample properties of the maximum likelihood estimator (MLE) and the QMLE of EGARCH processes using Monte Carlo methods. Linton (1997) proposed methods for bias reduction. The efficiency loss and/or bias of classical estimators result in poor estimates, unreliable forecasts and large confidence intervals. Thus, it is important to search for alternatives which do not assume a particular conditional distribution.

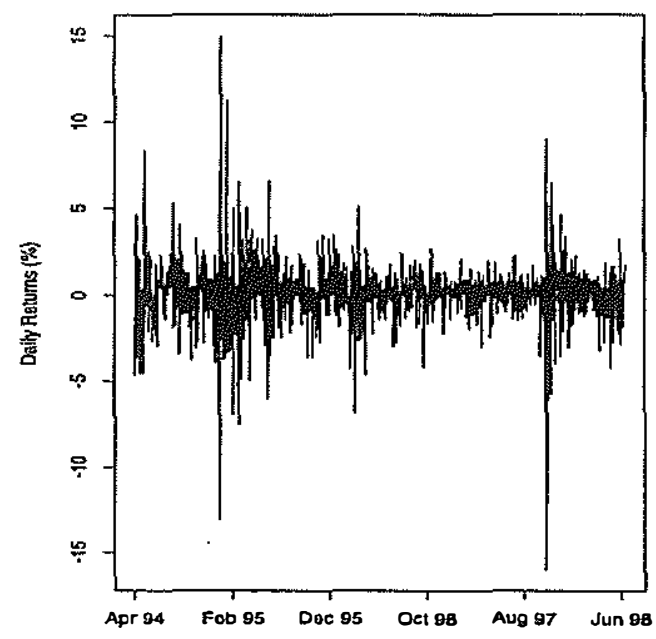

Figure 1

Daily returns of the Brazilian C-bond.

We introduce here a bias-robust and efficient estimation procedure resistant to outliers, which give more reliable estimates for (contaminated or not) GARCH processes. The new estimators are defined as the solutions to an optimization problem where the focus is to minimize a bounded function of the squared residuals standardized by a robust scale. This is the typical approach when obtaining the M-estimators and their extensions (Huber, 1981). We note that the objective function does not have to be a log-likelihood function. 
In the ARCH literature, related approaches focusing on the estimation of the conditional scale of the residuals rather than on the conditional variance already exist. For example, Koenker and Zhao (1996) proposed quantile regression methods for a class of ARCH models, and Schwert (1989) and Nelson and Foster (1994) proposed ARCH models for conditional scale under the assumption of normality.

This paper is organized as follows. In section 2 we define the constrained M-estimators for ARCH models. In sections 3 and 4 we obtain their robustness properties and show how to tune the estimators to obtain high efficiency under the core process. In section 5 we compare the bias of the classical and robust estimators using Monte Carlo methods. In section 6 we apply the robust procedure to estimate the volatility of four Brazilian series. Conclusions are given in section 7. All proofs may be found in the Appendix.

\section{Constrained M-Estimation for ARCH Models}

\subsection{The ARCH model and the classical estimators}

The Gaussian ARCH process of order $p$ assumes that

$$
x_{t} \mid \mathrm{x}_{t-1}^{-p+1} \sim \mathrm{N}\left(0, \sigma_{t}^{2}\right)
$$

for $t=1,2, \ldots, n$, where $\sigma_{t}^{2}=\alpha_{0}+\alpha_{1} x_{t-1}^{2}+\ldots+\alpha_{p} x_{t-p}^{2}$, and $\mathrm{x}_{t-1}^{-p+1}=\left(x_{t-1}, \ldots, x_{1}, x_{0}, \ldots, x_{-p+1}\right)$ represents the information available up to time $t$. Conditionally on the first $p$ observations, the QMLE $\left(\widehat{\alpha_{0}}, \widehat{\alpha_{1}}, \ldots, \widehat{\alpha_{p}}\right)$ of $\left(\alpha_{0}, \alpha_{1}, \ldots, \alpha_{p}\right)$ minimizes

$$
\frac{1}{n} \sum_{t=1}^{n} \frac{x_{t}^{2}}{2 \sigma_{t}^{2}}+\frac{1}{n} \sum_{t=1}^{n} \frac{1}{2} \log \sigma_{t}^{2}
$$


and is the solution of the $(p+1)$ first order estimating equations

$$
\begin{array}{r}
\frac{1}{n} \sum_{t=1}^{n} \tilde{\Psi}_{t}\left(\mathrm{x}_{t}^{-p+1},\left(\widehat{\alpha_{0}}, \widehat{\alpha_{1}}, \ldots, \widehat{\alpha_{p}}\right)\right)= \\
\frac{1}{n} \sum_{t=1}^{n} \frac{1}{2}\left(\frac{x_{t}^{2}}{\sigma_{t}^{2}}-1\right)\left(\frac{\mathbf{z}_{t}}{\sigma_{t}^{2}}\right)=\mathbf{0},
\end{array}
$$

where $\mathbf{z}_{t}^{\prime}=\left(1, x_{t-1}^{2}, x_{t-2}^{2}, \ldots, x_{t-p}^{2}\right)$, and $\tilde{\Psi}_{t}: \Re^{t+p} \times \Re^{p+1} \rightarrow \Re^{p+1}$.

The robustness measures of the QMLE were obtained in Mendes (1996) by expressing this estimator as a $\tilde{\Psi}$ estimator (Martin and Yohai, 1986). According to Hampel et al. (1986), an estimator may be considered robust if (i) it is highly efficient under a core process and reasonably efficient in a neighborhood of this process, (ii) it does not give distorted estimates in the presence of a few outliers, and (iii) it possess a bounded and continuous influence curve. In spite of the consistency and efficiency of maximum likelihood estimators under the correct specification, the QMLE of misspecified or contaminated ARCH processes lacks (i) (see Engle and González-Rivera, 1991); lacks (ii) (see section 5); and lacks (iii) (see Mendes, 1996). The next subsection formalizes the concepts of contamination neighborhoods and contaminated models.

\subsection{The general contamination model}

The serious effects of outliers on classical estimation procedures in general pass undetected by formal tests and analysis of residuals (Rousseeuw and Leroy, 1987). In the time series context, extra difficulties also arise due to the joint effect of the time configuration of the contaminating points (isolated, or in patches, or both) mixed with the structure of the model. To model the effect of innovations outliers and additive effects outliers (Fox, 1972) on ARCH processes 
we assume that the observations $y_{t}^{\gamma}$ follow the general replacement model (Martin and Yohai, 1986):

$$
y_{t}^{\gamma}=\left(1-z_{t}^{\gamma}\right) x_{t}+z_{t}^{\gamma} w_{t}
$$

where

$$
\begin{array}{ll}
x_{t} \sim \mu_{x}, & \text { is the ARCH core process, } \\
w_{t} \sim \mu_{w}, & \text { is a contaminating process, } \\
z_{t}^{\gamma} \sim \mu_{z}^{\gamma}, & \text { is a } 0-1 \text { process, }
\end{array}
$$

with $0 \leq \gamma \leq 1$ and $P\left(z_{t}^{\gamma}=1\right)=\gamma$, and where $y_{t}^{\gamma} \sim \mu_{y}^{\gamma}$ with $\mu_{y}^{0}=\mu_{x}$ in the case of no contamination.

The general replacement model may produce isolated and/or patches of outliers from the innovations and the additive models. The innovations outliers reflect the structure of the data generating process and may be obtained from (4) and (5), for example, by using a contaminating (conditional) distribution with heavier tails than the normal distribution.

The additive outliers (AO) model allows dependence between $x_{t}$ and $w_{t}$ and may be obtained from (4) by putting $w_{t}=x_{t}+v_{t} \Rightarrow$ $y_{t}^{\gamma}=x_{t}+z_{t}^{\gamma} v_{t}$, where $v$ has some specific distribution, and letting $z_{t}^{\gamma}$ be independent of $x_{t}$ and $v_{t}$.

The AO model seems to be a very realistic representation of various types of contamination occurring in practice. For example, isolated outliers may occur in stock markets as a result of news not confirmed in the next business day. This type of outliers may be obtained from the $\mathrm{AO}$ model, for example, by letting the $z_{t}^{\gamma}$ be i.i.d., and $w_{t}$ being a constant $\zeta$. Throughout the paper we shall work with the AO model. 


\subsection{The constrained M-estimators for ARCH models}

The M-estimators were proposed in Huber (1964) and may be considered one of the most important contributions to the robust theory. They may be understood as a generalization of the maximum likelihood estimator (Kent and Tyler, 1991) and, in the case of the ARCH models, this means to replace the objective function (2) by other loss function such as

$$
\frac{1}{n} \sum_{t=1}^{n} \rho\left(\frac{x_{t}^{2}}{\sigma_{t}^{2}}\right)+\frac{1}{n} \sum_{t=1}^{n} \frac{1}{2} \log \sigma_{t}^{2}
$$

where $\rho: \Re^{+} \rightarrow \Re^{+}$is a monotone nondecreasing function with a unique minimum at zero. If $\rho$ is differentiable, the solutions to (refeq10) must satisfy the system of estimating equations

$$
\frac{1}{n} \sum_{t=1}^{n}\left(2 u\left(\frac{x_{t}^{2}}{\sigma_{t}^{2}}\right)\left(\frac{x_{t}^{2}}{\sigma_{t}^{2}}\right)-1\right)\left(\frac{\mathrm{z}_{t}}{\sigma_{t}^{2}}\right)=\mathbf{0},
$$

obtained by taking the derivatives of (6) with respect to $\left(\alpha_{0}, \ldots, \alpha_{p}\right)$, and where $u(s)=\partial \rho(s) / \partial s$.

Notice that (2) and (3) may be obtained from (6) and (7) by taking $\rho(s)=\frac{1}{2} s$. Better results in terms of robustness against the influence of outliers may be obtained if we assume $\rho(\cdot)$ bounded. In this case $u(\cdot)$ is redescending, i.e., $u(s) \rightarrow 0$ as $s \rightarrow \infty$. We assume that $\exists 0<r<\infty$ such that $u(s)=0$ for $s \geq r$. We would like to observe that this procedure may be viewed as the first step in the robustification of the classical estimates for ARCH models. A possible second step (that we will address in future research) is to also bound the parcel $\frac{1}{n} \sum \frac{1}{2} \log \sigma_{t}^{2}$ by means of some weight function.

It turns out that, when $\rho(\cdot)$ is bounded, it is not meaningful to minimize (refeq10) unconditionally. To bound the scale $\sigma_{t}^{2}$ away from 
zero, the constrained M-estimators (Kent and Tyler, 1996, Mendes and Tyler, 1996), or CM-estimators for short, introduce the restriction

$$
\frac{1}{n} \sum_{t=1}^{n} \rho\left(\frac{x_{t}^{2}}{\sigma_{t}^{2}}\right) \leq \epsilon \rho(\infty),
$$

where $0 \leq \epsilon \leq 1$ is a fixed number. The introduction of this constrain will produce estimators that possess good local robustness properties at the core ARCH process and have a better chance to resist to outliers. Likewise the CM-estimators proposed for other statistical models, whenever the solutions are obtained under equality constrain they correspond to an S-estimator. For details concerning the concept of CM-estimation and its relationship to the concept of S-estimation we refer the reader to Kent and Tyler (1996).

The CM-estimators $\left(\mathrm{T}_{n}\right)$ for $\mathrm{ARCH}(p)$ models may be expressed as $\tilde{\Psi}$ estimators (Martin and Yohai, 1986). This means that they satisfy a system of equations

$$
\frac{1}{n} \sum_{t=1}^{n} \tilde{\Psi}_{t}\left(\mathbf{x}_{t}^{-p+1}, \mathbf{T}_{n}\right)=\mathbf{0},
$$

where the function $\tilde{\Psi}_{t}: \Re^{t+p} \times \Re^{p+1} \rightarrow \Re^{p+1}$ is bounded. More specifically, the CM-estimators satisfy

$$
\frac{1}{n} \sum_{t=1}^{n}\left(2 u\left(\frac{x_{t}^{2}}{\sigma_{t}^{2}}\right) \frac{x_{t}^{2}}{\sigma_{t}^{2}}\right)\left(\frac{z_{t}}{\sigma_{t}^{2}}\right)-\left(\frac{\frac{1}{n} \sum_{t=1}^{n} 2 u\left(\frac{x_{t}^{2}}{\sigma_{t}^{2}}\right) \frac{x_{t}^{2}}{\sigma_{t}^{2}} \frac{1}{\sigma_{t}^{2}}}{\frac{1}{n} \sum_{t=1}^{n} \frac{1}{\sigma_{t}^{2}}}\right) \frac{1}{n} \sum_{t=1}^{n}\left(\frac{x_{t}}{\sigma_{t}^{2}}\right)=0
$$

where the first equation in the system of $(p+1)$ equations $(10)$ is either replaced by

$$
\frac{1}{n} \sum_{t=1}^{n} 2 u\left(\frac{x_{t}^{2}}{\sigma_{t}^{2}}\right) \frac{x_{t}^{2}}{\sigma_{t}^{2}} \frac{1}{\sigma_{t}^{2}}-\frac{1}{n} \sum_{t=1}^{n} \frac{1}{\sigma_{t}^{2}}=0
$$


in the case of strict inequality constrain, when they correspond to the critical points of the objective function (6); or it is replaced by

$$
\frac{1}{n} \sum_{t=1}^{n} \rho\left(\frac{x_{t}^{2}}{\sigma_{t}^{2}}\right)-\epsilon \rho(\infty)=0
$$

under equality constrain. In the latter case they are the Lagrange multiplier solutions to the equality constrained minimization problem.

In the following section we obtain some robustness measures for the the CM-estimators for ARCH models.

\section{The Robustness Properties of the CM-Estimatores}

To obtain the robustness properties of the CM-estimators $\mathbf{T}_{n}$ it is convenient to define first their asymptotic version, the CMfunctionals $(\mathbf{T})$. To obtain the related asymptotic theory we first assume.

Condition 3.1. For $s>0, \rho(s)$ is bounded, continuous, and strictly increasing for $\rho(s)<\rho(\infty)$. In addition, $\rho(0)=0$.

Under suitable regularity conditions (Martin and Yohai, 1986), the CM-functionals defined through a $\rho$ function satisfying Condition 3.1 , are the solutions to the system of $(p+1)$ equations

$$
\int \tilde{\Psi}\left(\mathbf{x}_{1}, \mathbf{T}\right) d \mu\left(\mathbf{x}_{1}\right)=\mathbf{0},
$$

where the limit function $\bar{\Psi}$ satisfies for each $\mathbf{t} \in \Re^{p+1}$

$$
\lim _{t \rightarrow \infty} \tilde{\Psi}_{t}\left(a_{t}, a_{t-1}, \ldots, a_{-p+1}, \mathbf{t}\right)=\tilde{\Psi}(\mathbf{a}, \mathbf{t}) \quad \forall \mathbf{a}=\left(a_{1}, a_{2}, \ldots\right) \in \Re^{\infty} .
$$


Here, $\mathbf{x}_{1}$ represent dummy arguments, possible since we work with stationary measures, and $\mathbf{z}_{1}^{\prime}=\left(1, x_{0}^{2}, x_{-1}^{2}, \ldots, x_{-p+1}^{2}\right)$. We denote by $\mathbf{t}_{0}=\mathbf{T}(\mu)$ the unique root of (13) at $\mu=\mu_{x}$, and assume that the CM-functionals are defined not just for $\mu_{x}$ but also for the contamination measures $\mu_{y}^{\gamma}$. It is assumed that there exists a subset of all stationary measures for which the expectation in (13) exists and is finite.

For the CM-functionals the estimating equations are given by

$$
\int\left(\left(2 u\left(\frac{x_{1}^{2}}{\sigma_{1}^{2}}\right) \frac{x_{1}^{2}}{\sigma_{1}^{2}}\right)\left(\frac{x_{1}}{\sigma_{1}^{2}}\right)-\left(\frac{\int 2 u\left(\frac{x_{1}^{2}}{\sigma_{1}^{2}}\right) \frac{x_{1}^{2}}{\sigma_{1}^{2}} \frac{1}{\sigma_{1}^{2}} d \mu\left(\mathbf{x}_{1}\right)}{\int \frac{1}{\sigma_{1}^{2}} d \mu\left(\mathbf{x}_{1}\right)}\right)\left(\frac{z_{1}}{\sigma_{1}^{2}}\right)\right) d \mu\left(\mathbf{x}_{1}\right)=0
$$

where either we have

$$
\int 2 u\left(\frac{x_{1}^{2}}{\sigma_{1}^{2}}\right) \frac{x_{1}^{2}}{\sigma_{1}^{2}} \frac{1}{\sigma_{1}^{2}} d \mu\left(\mathrm{x}_{1}\right)=\int \frac{1}{\sigma_{1}^{2}} d \mu\left(\mathbf{x}_{1}\right)
$$

under strict inequality constrain, or the first entry of (14) is replaced by

$$
\int \rho\left(\frac{x_{1}^{2}}{\sigma_{1}^{2}}\right) d \mu\left(\mathbf{x}_{1}\right)=\epsilon \rho(\infty)
$$

under equality constrain.

Under the core Gaussian process the random variable $x_{1}^{2} / \sigma_{1}^{2}$ has conditionally a chi-square distribution with one degree of freedom. Let $S=x_{1}^{2} / \sigma_{1}^{2}$ and let $\lambda_{L}$ represent the unique solution of $\mathrm{E} \rho(S / \lambda)=$ $\epsilon \rho(\infty)$, the asymptotic version of the constrain. By the definition of the CM-estimators and Condition 3.1, we conclude that the CMfunctionals minimize

$$
\mathrm{E} \rho\left(\frac{S}{\lambda}\right)+\frac{1}{2} \log \lambda
$$

subject to

$$
\lambda \geq \lambda_{L}
$$


The tuning constant $\lambda$ is used to tune the CM-estimators for good local properties at the Gaussian process.

Asymptotic properties obtained in Martin and Yohai (1986) for the $\tilde{\boldsymbol{\Psi}}$ estimators, and in Kent and Tyler (1996) for the CMestimators also hold in the case of ARCH models. It can be shown that the CM-estimators are continuous, Fisher consistent, and asymptotically normally distributed. We leave this problem open, but it may not be difficult to obtain these results by applying the maximum likelihood approach used by Weiss (1986) in section 3. Under the assumption that the process have finite fourth moment, the existence of the CM-estimators may be proved by showing that the limit of the objective function (6) as $n \rightarrow \infty$ exists almost sure for all $\mathbf{T}_{n}$ in the parameter space, and is uniquely minimized at $t_{0}$. Then, consistency and asymptotic normality can be proved following the lines of the proof of Theorem 3.2 and Theorem 3.3 of Weiss (1986) and the results found in Basawa, Feigin and Heyde (1976). This means that the CM-estimators $\mathbf{T}_{\boldsymbol{n}}$ converge almost sure to their asymptotic value $\mathbf{T}$ which estimates the true parameters $\boldsymbol{\alpha}$ multiplied by the constant $\lambda$, that is, $n^{1 / 2}\left(\mathrm{~T}_{n}-\mathrm{T}\right) \rightarrow \mathrm{N}\left(0, \mathrm{~V}\left(\tilde{\Psi}, \mu_{x}\right)\right)$. To obtain the asymptotic covariance matrix and other asymptotic properties of the CM-estimators we assume

Condition 3.2. The function $\rho$ has a continuous second derivative $u^{\prime}(s)$, and $u(s), u^{\prime}(s), s u(s)$ and $s u^{\prime}(s)$ are bounded. The function $\tilde{\boldsymbol{\Psi}}$ is odd in each variable, and

(i) $\mathbf{E} \tilde{\boldsymbol{\Psi}}\left(\mathbf{x}_{1}, \mathbf{t}\right)$ is differentiable at $\mathbf{t}=\mathbf{t}_{0}$.

(ii) $\mathbf{C}=\mathrm{E}\left[\frac{\partial}{\partial \mathrm{t}} \tilde{\boldsymbol{\Psi}}\left(\mathbf{x}_{1}, \mathrm{t}\right)\right]_{\mathrm{t}=\mathrm{t}_{0}}$ exists and is nonsingular.

(iii) $\mathrm{E}\left[\tilde{\boldsymbol{\Psi}}\left(\mathbf{x}_{1}, \mathrm{t}\right) \tilde{\Psi}^{\prime}\left(\mathbf{x}_{1}, \mathrm{t}\right)\right]_{\mathbf{t}=\mathrm{t}_{0}}$ exists and is nonsingular.

(iv) $\mathrm{E}\left[\frac{z_{1}}{\sigma_{1}^{2}} \frac{z_{1}^{\prime}}{\sigma_{1}^{2}}\right]$ under the core process exists and is nonsingular.

Revista de Econometria 19 (1) Maio 1999 
We first compute for the CM-estimators the Hampel's (1974) concept of influence curve (ICH), originally proposed for i.i.d. observations. Let $\mu_{\gamma}=(1-\gamma) \mu_{x}+\gamma \delta_{\mathbf{y}_{1}}$ be a measure associated with a contaminated process, where $\delta_{\mathbf{y}_{1}}$ represents a unit mass at $\mathbf{y}_{1} \in \Re^{\infty}$, and $0 \leq \gamma \leq 1$. The ICH for time series $\tilde{\boldsymbol{\Psi}}$ estimators is defined as

$$
\operatorname{ICH}\left(\mathbf{y}_{1}, \mathbf{T}, \mu\right)=\lim _{\gamma \rightarrow 0} \frac{\mathbf{T}\left(\mu_{\gamma}\right)-\mathbf{T}(\mu)}{\gamma},
$$

for all $\mathrm{y}_{1} \in \Re^{\infty}$ for which this limit exists. Given Conditions 3.1 and 3.2 and under regularity conditions, the $\mathbf{I C H}\left(\mathrm{y}_{1}\right)$ for a functional $\mathbf{T}$ at the core process $\mu_{x}$ may be computed using the relation

$$
\operatorname{ICH}\left(\mathbf{y}_{1}, \mathbf{T}, \mu_{x}\right)=-\mathbf{C}^{-1} \tilde{\boldsymbol{\Psi}}\left(\mathbf{y}_{1} ; \mathbf{t}_{0}\right) .
$$

The influence curve of the CM-estimators for ARCH models is given in Theorem 3.1.

Theorem 3.1. Assume that the CM-functionals are defined by (13) for the nominal process with measure $\mu_{x}$ and for the contaminating measure $\mu_{\gamma}$. The influence curves of the CM-estimators for ARCH models are given by

A. Under strict inequality constrain

$$
\begin{aligned}
& \operatorname{ICH}\left(\mathbf{y}_{1}, \mathbf{T}, \mu_{x}\right)= \\
& =B\left(\tilde{\boldsymbol{\Psi}} ; \mathbf{t}_{0}\right)^{-1}[M(\boldsymbol{\alpha})]^{-1}\left(2 u\left(\frac{y_{1}^{2}}{\sigma_{1}^{2}}\right)\left(\frac{y_{1}^{2}}{\sigma_{1}^{2}}\right)-1\right)\left(\frac{\mathbf{z}_{1}^{*}}{\sigma_{1}^{2}}\right),
\end{aligned}
$$

B. Under equality constrain

$$
\begin{aligned}
& \operatorname{ICH}\left(\mathrm{y}_{1}, \mathrm{~T}, \mu_{x}\right)= \\
& \mathbf{B}\left(\tilde{\boldsymbol{\Psi}} ; \mathbf{t}_{0}\right)^{-1}[M(\alpha)]^{-1}\left[\begin{array}{l}
\left(\left(\rho\left(\frac{y_{1}^{2}}{\sigma_{1}^{2}}\right)-\epsilon \rho(\infty)\right) \sigma_{1}^{2}\right)\left(\frac{1}{\sigma_{1}^{2}}\right) \\
\left(2 u\left(\frac{y_{1}^{2}}{\sigma_{1}^{2}}\right)\left(\frac{y_{1}^{2}}{\sigma_{1}^{2}}\right)-A\right)\left(\frac{\mathbf{y}_{1}^{*}}{\sigma_{1}^{2}}\right)
\end{array}\right],
\end{aligned}
$$


where $\mathbf{z}_{1}^{*}=\left(1, \mathbf{y}_{1}^{*}\right)^{\prime}=\left(1, y_{0}^{2}, y_{-1}^{2}, \ldots, y_{-p+1}^{2}\right)^{\prime}, M(\boldsymbol{\alpha})=\mathrm{E}\left[\left(\mathbf{z}_{1} / \sigma_{1}^{2}\right)\right.$ $\left.\left(\mathbf{z}_{1}^{\prime} / \sigma_{1}^{2}\right)\right], B\left(\tilde{\boldsymbol{\Psi}} ; \mathbf{t}_{0}\right)=\mathrm{E}[-b(\tilde{\boldsymbol{\Psi}})]$ at $\mathbf{t}_{0}, \quad b(\tilde{\boldsymbol{\Psi}})=(-2) u^{\prime}\left(x_{1}^{2} / \sigma_{1}^{2}\right)\left(x_{1}^{2} / \sigma_{1}^{2}\right)^{2}$ $-4 u\left(x_{1}^{2} / \sigma_{1}^{2}\right)\left(x_{1}^{2} / \sigma_{1}^{2}\right)+1, \mathrm{~B}\left(\tilde{\Psi} ; \mathbf{t}_{0}\right)=\mathrm{E}[-\mathbf{b}(\tilde{\Psi})]$ at $\mathbf{t}_{0}, \mathbf{b}(\tilde{\Psi})$ is a diagonal matrix with diagonal $\left(-u\left(x_{1}^{2} / \sigma_{1}^{2}\right)\left(x_{1}^{2} / \sigma_{1}^{2}\right) \sigma_{1}^{2}, b^{*}, b^{*}, \ldots, b^{*}\right)$, where $b^{*}=-2 u^{\prime}\left(x_{1}^{2} / \sigma_{1}^{2}\right)\left(x_{1}^{2} / \sigma_{1}^{2}\right)^{2}-4 u\left(x_{1}^{2} / \sigma_{1}^{2}\right)\left(x_{1}^{2} / \sigma_{1}^{2}\right)+A$, and $A=\left[\int 2 u\left(\frac{x_{1}^{2}}{\sigma_{1}^{2}}\right) \frac{x_{1}^{2}}{\sigma_{1}^{2}} \frac{1}{\sigma_{1}^{2}} d \mu\left(\mathrm{x}_{1}\right)\right] /\left[\int \frac{1}{\sigma_{1}^{2}} d \mu\left(\mathrm{x}_{1}\right)\right]$.

Proof. See Appendix.

The asymptotic covariance $\mathrm{V}\left(\tilde{\boldsymbol{\Psi}}, \boldsymbol{\mu}_{x}\right)$ of the CM-estimators under the nominal Gaussian process and the efficiency relative to the maximum likelihood estimator are easily obtained using the results in Martin and Yohai (1986) and in Mendes (1996).

Theorem 3.2. Under regularity conditions the asymptotic variancecovariance matrix of the CM-estimators for $\mathrm{ARCH}$ models at the core Gaussian process $\mu_{x}$ is given by

A. Under strict inequality constrain

$$
\begin{aligned}
& \mathrm{V}\left(\tilde{\boldsymbol{\Psi}}, \mu_{x}\right)= \\
& \frac{1}{B^{2}\left(\tilde{\boldsymbol{\Psi}} ; \mathrm{t}_{0}\right)} \mathrm{E}\left[4 u^{2}\left(\frac{x_{1}^{2}}{\sigma_{1}^{2}}\right)\left(\frac{x_{1}^{2}}{\sigma_{1}^{2}}\right)^{2}-4 u\left(\frac{x_{1}^{2}}{\sigma_{1}^{2}}\right)\left(\frac{x_{1}^{2}}{\sigma_{1}^{2}}\right)+1\right][M(\alpha)]^{-1}(21)
\end{aligned}
$$

B. Under equality constrain

$$
\mathrm{V}\left(\tilde{\Psi}, \mu_{x}\right)=\mathrm{B}\left(\tilde{\Psi} ; \mathrm{t}_{0}\right)^{-2} \mathrm{E}[\mathrm{Z}][M(\alpha)]^{-2}
$$

where $\mathbf{Z}$ is $a(p+1) \times(p+1)$ symmetric matrix, $\mathbf{Z}=\mathbf{v}^{\prime} \mathbf{v}$, with $\mathbf{v}=\left(\left(\rho\left(x_{1}^{2} / \sigma_{1}^{2}\right)-\epsilon \rho(\infty)\right),\left(2 u\left(x_{1}^{2} / \sigma_{1}^{2}\right)\left(x_{1}^{2} / \sigma_{1}^{2}\right)-A\right)\left(x_{0}^{2} / \sigma_{1}^{2}\right)\right.$

Revista de Econometria 19 (1) Maio 1999 
$\left., \ldots,\left(2 u\left(x_{1}^{2} / \sigma_{1}^{2}\right)\left(x_{1}^{2} / \sigma_{1}^{2}\right)-A\right)\left(x_{-p+1}^{2} / \sigma_{1}^{2}\right)\right)$. All other quantities are as previously defined.

Proof. See Appendix.

Under strict inequality constrain, the asymptotic efficiency relative to the maximum likelihood estimator is the ratio

$$
\frac{2 B^{2}\left(\tilde{\Psi} ; \mathrm{t}_{0}\right)}{\mathrm{E}\left[4 u^{2}\left(\frac{x_{1}^{2}}{\sigma_{1}^{2}}\right)\left(\frac{x_{1}^{2}}{\sigma_{1}^{2}}\right)^{2}-4 u\left(\frac{x_{1}^{2}}{\sigma_{1}^{2}}\right) \frac{x_{1}^{2}}{\sigma_{1}^{2}}+1\right]},
$$

and under equality constrain it is equal to $2[\mathrm{E}(\mathrm{Z})]^{-1} \mathbf{B}^{2}\left(\tilde{\Psi} ; \mathrm{t}_{0}\right) M(\boldsymbol{\alpha})$.

In section 4 we show that the CM-estimators may be tuned to possess high efficiency at the nominal process, and we exemplify the tuning procedure by computing (22) using the Biweighted Tukey $\rho$ function.

The influence functional (IF) is a more general concept which includes Hampel's definition of influence curve based on point mass contamination as a particular case. It was introduced by Martin and Yohai (1986) and takes into account the time-configuration of the contaminating points. The influence functional of a time series functional $\mathbf{T}$ is defined as

$$
\mathbf{I F}\left(\mu_{w} \mathbf{T}\{\gamma\}\right)=\lim _{\gamma \rightarrow 0} \frac{\mathbf{T}\left(\mu_{y}^{\gamma}\right)}{\gamma},
$$

if this limit exists, and depend upon a particular trajectory $\left\{\mu_{y}^{\gamma}\right\}$ of contamination measures. Under certain conditions (see Martin and Yohai, 1986, for details) the relation $\operatorname{IF}\left(\mu_{w}, \mathbf{T},\left\{\mu_{y}^{\gamma}\right\}\right)=$ $\lim _{\gamma \rightarrow 0}\left\{\operatorname{E~} \operatorname{ICH}\left(y_{1}^{\gamma}\right) / \gamma\right\}$ holds and it is used in Theorem 4.1 to compute the expression of the CM-influence functionals for ARCH models. Since we are dealing with AO model, we assume that the process 
$\left\{x_{i}, w_{i}\right\}$ is independent of the process $\left\{z_{i}^{\gamma}\right\}$ with measure $\mu_{z}^{k, \gamma}$, which may generate patches of length $k$ of outliers in model (4), and we use the notation $\operatorname{IF}_{\mathrm{AO}, k}\left(\mu_{v}, \mathrm{~T}, \boldsymbol{\alpha}\right)$.

Theorem 3.3. Assume the $A O$ model, and suppose that $\mu_{x w z}=$ $\mu_{x v} \mu_{z}^{k, \gamma}=\mu_{x} \mu_{v} \mu_{z}^{k, \gamma}$ The influence functionals $\operatorname{IF}_{\mathrm{AO}, k}\left(\mu_{v}, \mathbf{T}, \boldsymbol{\alpha}\right)$ of the constrained $M$-functionals for $A R C H$ models (T) are given by:

A. Under strict inequality constrain:

- For independent outliers $(k=1)$

$$
\operatorname{IF}_{\mathrm{AO}, 1}\left(\mu_{v}, \mathbf{T}, \boldsymbol{\alpha}\right)=\frac{[M(\boldsymbol{\alpha})]^{-1}}{B\left(\tilde{\boldsymbol{\Psi}} ; \mathbf{t}_{0}\right)} \mathrm{E}\left[\left(2 u\left(\frac{v_{1}^{2}}{\sigma_{1}^{2}}\right)\left(\frac{v_{1}^{2}}{\sigma_{1}^{2}}\right)-1\right)\left(\frac{\mathbf{z}_{1}}{\sigma_{1}^{2}}\right)\right]
$$

where $M(\boldsymbol{\alpha})$ and $B\left(\tilde{\boldsymbol{\Psi}} ; \mathrm{t}_{0}\right)$ are as previously defined.

- For patches of outliers of length $k \geq 2$

$$
\operatorname{IF}_{\mathrm{AO}, k}\left(\mu_{v}, \mathbf{T}, \boldsymbol{\alpha}\right)=\frac{[M(\boldsymbol{\alpha})]^{-1}}{k B\left(\tilde{\Psi} ; \mathbf{t}_{0}\right)} \sum_{j=0}^{k-1} \mathrm{E}\left[\left(2 u\left(\frac{v_{1}^{2}}{\sigma_{1}^{2}}\right)\left(\frac{v_{1}^{2}}{\sigma_{1}^{2}}\right)-1\right)\left(\frac{\mathbf{z}_{1}^{(j)}}{\sigma_{1}^{2}}\right)\right]
$$

where $\mathbf{z}_{1}^{(j)}=\left(1, v_{0}^{2}, v_{-1}^{2}, \ldots, v_{1-j}^{2}, x_{-j}^{2}, \ldots, x_{-q+1}^{2}\right)^{\prime}$.

B. Under equality constrain:

- For independent outliers $(k=1)$ the $\operatorname{IF}_{\mathrm{AO}, 1}\left(\mu_{v}, \mathbf{T}, \boldsymbol{\alpha}\right)$ is given by

$$
[M(\alpha)]^{-1} \mathbf{B}\left(\tilde{\boldsymbol{\Psi}} ; \mathbf{t}_{0}\right)^{-1} \mathrm{E}\left[\left(\begin{array}{l}
\left(\rho\left(\frac{v_{1}^{2}}{\sigma_{1}^{2}}\right)-\epsilon \rho(\infty)\right) \sigma_{1}^{2} \\
2 u\left(\frac{v_{1}^{2}}{\sigma_{1}^{2}}\right)\left(\frac{v_{1}^{2}}{\sigma_{1}^{2}}\right)-A
\end{array}\right)\left(\frac{\mathbf{z}_{1}}{\sigma_{1}^{2}}\right)\right]
$$

Revista de Econometria 19 (1) Maio 1999 
- For patches of outliers of length $k \geq 2$ the $\mathrm{IF}_{\mathrm{AO}, k}\left(\mu_{v}, \mathbf{T}, \boldsymbol{\alpha}\right)$ is given by

$$
\frac{1}{k}[M(\boldsymbol{\alpha})]^{-1} \mathbf{B}\left(\tilde{\Psi} ; \mathbf{t}_{0}\right)^{-1} \sum_{j=0}^{k-1} \mathrm{E}\left[\left(\begin{array}{l}
\left(\rho\left(\frac{v_{1}^{2}}{\sigma_{1}^{2}}\right)-\epsilon \rho(\infty)\right) \sigma_{1}^{2} \\
2 u\left(\frac{v_{1}^{2}}{\sigma_{1}^{2}}\right)\left(\frac{v_{1}^{2}}{\sigma_{1}^{2}}\right)-A
\end{array}\right)\left(\frac{\mathbf{z}_{1}^{(j)}}{\sigma_{1}^{2}}\right)\right]
$$

where all quantities have already been defined.

Proof. See Appendix.

Under strict inequality, it is very interesting to observe the role of the bounded redescending function $u(\cdot)$ taking care of the effect of a single outlier, and the role of the $\mathrm{ARCH}$ structure also bounding the influence of a sequence of outliers. This means that if $k>1$ the ARCH structure takes advantage of the "temporal clustering of outliers, and minimize their effects". This approach taken by the ARCH model was predicted in Engle (1982), page 992.

Martin and Yohai (1986) define the gross error sensitivity (GES) of a functional $\mathbf{T}$ at a family $\mathbf{P}$ of $\operatorname{arcs}\left\{\mu_{y}^{\gamma}\right\},\left\{\mu_{y}^{\gamma}\right\}=\left\{\mu_{y}^{\gamma}: 0 \leq \gamma<\right.$ $1\}$, as

$$
\operatorname{GES}(\mathbf{P}, \mathbf{T})=\sup _{\left\{\mu_{y}^{\gamma}\right\} \in \mathbf{P}}\left|\operatorname{IF}\left(\mu_{w}, \mathbf{P},\left\{\mu_{y}^{\gamma}\right\}\right)\right|
$$

For the CM-functionals, the GES for isolated and patches of outliers are the supremum of the absolute value of each coordinate of (23)(26).

In the AO model a very special case of the influence functionals arises when $\mathrm{P}\left(v_{t}=\zeta\right)=1, \zeta$ a constant (see subsection 2.2). In this case $\mathbf{P}=\left\{\delta_{\zeta}\right\}$, where $\delta_{\zeta}$ represents the point mass on $\Re^{\infty}$, and the GES is simply the supremum of the influence functional $\operatorname{IF}_{A O, 1}(\zeta)$ 
over all $\zeta$. For example, for the CM-estimators under strict inequality constrain, when $k=1$ and $\mathbf{P}=\left\{\delta_{\zeta}\right\}$, the $\operatorname{GES}_{A O, 1}$ is the supremum over all $\zeta$ of the absolute value of

$$
\frac{1}{B\left(\tilde{\Psi} ; \mathbf{t}_{0}\right)} \mathrm{E}\left[\left(2 u\left(\frac{\zeta^{2}}{\sigma_{1}^{2}}\right)\left(\frac{\zeta^{2}}{\sigma_{1}^{2}}\right)-1\right)\left(\frac{\mathbf{z}_{1}}{\sigma_{1}^{2}}\right)\right],
$$

multiplied by a constant matrix. Note that the form of the influence functionals are independent of the order $p$ of the ARCH process, which affects just the number of coordinates. We show in the next section that, given a $\rho$ function, the IF and therefore the GES depend upon a constant $\lambda$, which may be chosen such that the resulting CM-estimators have a small GES.

\section{Tuning the CM-Estimators}

The CM-estimators may be tuned by the introduction of a multiplicative constant $c$ and a corresponding constant $\lambda$ in the definition of the $\rho$ function (see Kent and Tyler, 1996, for details). To illustrate the tuning process for the CM-estimators for Gaussian ARCH models we now set the $\epsilon$ in equation (8) equal to $1 / 2$ and select the Biweighted $\rho$ function defined as

$$
c \rho\left(\frac{S}{\lambda}\right)=c \begin{cases}3 \frac{S}{\lambda}-3\left(\frac{S}{\lambda}\right)^{2}+\left(\frac{S}{\lambda}\right)^{3} & \text { if } \frac{S}{\lambda} \leq 1 \\ 1 & \text { if } \frac{S}{\lambda}>1\end{cases}
$$

where $S=x_{1}^{2} / \sigma_{1}^{2}$ is as previously defined. The corresponding Biweighted $\tilde{\boldsymbol{\Psi}}$ function under strict inequality is

$$
\begin{aligned}
\tilde{\Psi}_{c}\left(\mathbf{x}_{1}, \mathbf{T}, \lambda\right) & =\tilde{\Psi}_{c}\left(\frac{S}{\lambda}\right) \\
& =\left[6 c\left(\frac{S}{\lambda}-2\left(\frac{S}{\lambda}\right)^{2}+\left(\frac{S}{\lambda}\right)^{3}\right) \mathbf{I}_{\left(\frac{S}{\lambda} \leq 1\right)}-1\right]\left(\frac{\mathbf{z}_{1}}{\lambda \sigma_{1}^{2}}\right),
\end{aligned}
$$

Revista de Econometria 19 (1) Maio 1999 
where $\mathbf{I}$ is the indicator function. In this section we change slightly the notation to represent the effects of the tuning constant $c$ and the value $\lambda$. The constant $c$ is an increasing function of $\lambda$ and given a value for $\lambda$, for this choice of $\rho$ function the constant $c$ is calculated (see (14) and (15)) using

$$
c=\frac{1}{\mathrm{E}\left[\left(6 \frac{S}{\lambda}-12\left(\frac{S}{\lambda}\right)^{2}+6\left(\frac{S}{\lambda}\right)^{3}\right) \mathbf{I}_{\left(\frac{S}{\lambda} \leq 1\right)}\right]} .
$$

For the Biweighted CM-estimators the expression for their influence curve in the strict inequality case is

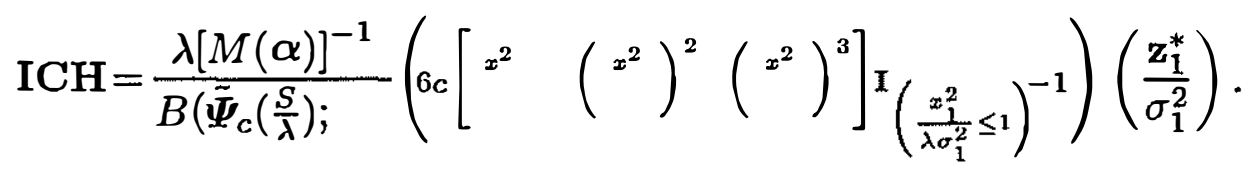

The asymptotic efficiency of the Biweighted CM-estimators (under strict inequality constrain) for Gaussian ARCH models, relative to the MLE (see Mendes, 1996), computed using expression (22), is

$$
2\left[B\left(\tilde{\Psi}_{c}\left(\frac{S}{\lambda}\right) ; \mathbf{t}_{0}\right)\right]^{2}
$$

$c^{2} 36($

where $B\left(\tilde{\Psi}_{c}(S / \lambda)\right.$;

$\mathrm{e}^{r}(\lambda)$ represents the conditional expectation $\mathrm{E}\left[(S / \lambda)^{r} \mathrm{I}_{(S \leq \lambda)}\right], r=$ $0,1,2, \ldots$ (see computations in Mendes, 1995).

Table 1 shows the asymptotic efficiency relative to the Gaussian maximum likelihood estimator, for a selection of values of $\lambda$. For the Biweighted $\rho$ function the value of $\lambda_{L}$ is $2.3952(c=2.5684)$. 
Beatriz V.M. Mendes and A.M. Duarte Júnior

Table 1

The Efficiency and GES of the Biweighted CM-Estimators

\begin{tabular}{cccc}
\hline$\lambda$ & $c$ & $\begin{array}{c}\text { Efficiency relative } \\
\text { to the MLE }\end{array}$ & GES \\
\hline 14.4 & 3.862 & 0.702 & 3.862 \\
20.5 & 4.598 & 0.850 & 4.397 \\
24.6 & 4.882 & 0.897 & 4.882 \\
26.2 & 5.084 & 0.910 & 5.084 \\
\hline
\end{tabular}

The influence functionals $\mathbf{I F}_{A O, 1}$ and $\mathrm{IF}_{A O, k}$ under strict inequality for the Biweighted CM-estimators for Gaussian ARCH models are respectively

$$
\left.\frac{\lambda M(\boldsymbol{\alpha})^{-1}}{B\left(\tilde{\Psi}_{c}\left(\frac{S}{\lambda}\right) ; \mathbf{t}_{0}\right)} \mathrm{E}\left[\left(6 c\left[\frac{v_{1}^{2}}{\lambda \sigma_{1}^{2}}-2\left(\frac{v_{1}^{2}}{\lambda \sigma_{1}^{2}}\right)^{2}+\left(\frac{v_{1}^{2}}{\lambda \sigma_{1}^{2}}\right)^{3}\right] \mathrm{I}\left(\frac{v_{1}^{2}}{\lambda \sigma_{1}^{2}} \leq 1\right)\right)^{-1}\right)\left(\frac{z_{1}}{\sigma_{1}^{2}}\right)\right]
$$

and

$$
\frac{\lambda M(\alpha)^{-1}}{k B\left(\tilde{\Psi}_{c}\left(\frac{S}{\lambda}\right) ; \mathrm{t}_{0}\right)} \mathrm{E}\left[\left(6 c\left[\frac{v_{1}^{2}}{\lambda \sigma_{1}^{2}}-2\left(\frac{v_{1}^{2}}{\lambda \sigma_{1}^{2}}\right)^{2}+\left(\frac{v_{1}^{2}}{\lambda \sigma_{1}^{2}}\right)^{3}\right] \mathrm{I}_{\left(\frac{v_{1}^{2}}{\lambda \sigma_{1}^{2}} \leq 1\right)}-1\right)\left(\frac{z_{1}^{(j)}}{\sigma_{1}^{2}}\right)\right]
$$

As we have already commented, an special case of the influence functionals, which capture the notion of influence (well expressed by the $\mathbf{I C H}$ in the i.i.d. case), is the $\operatorname{IF}_{A O, 1}(\zeta)$ for $\zeta \in \Re$. We plot in Figure 2 these quantities against the contaminating value $\zeta$, for the QMLE and for the Biweighted CM-estimators. In this figure the tuning constant $\lambda$ was set equal to 26 resulting in $91 \%$ efficient estimates under the core Gaussian process. 


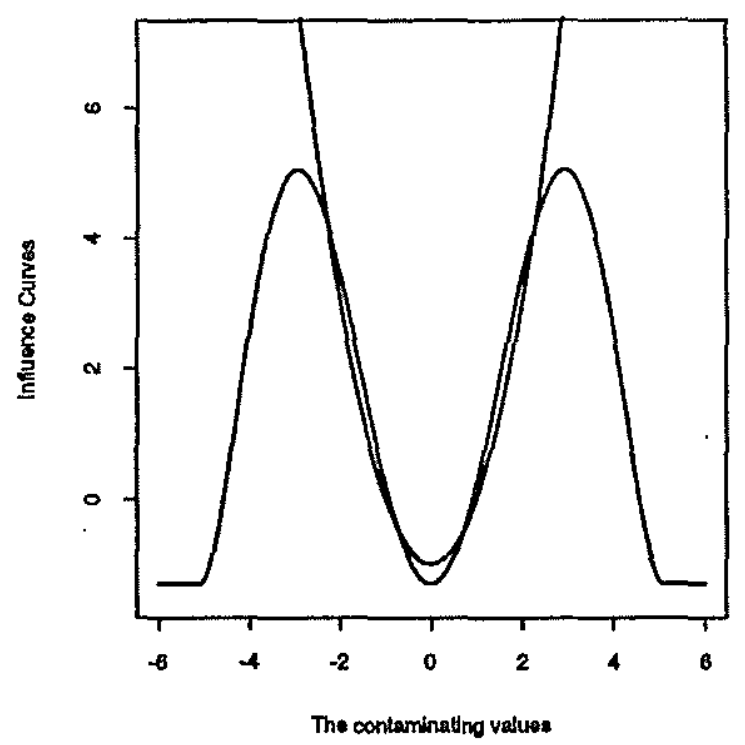

Figure 2

The CM and the QMLE influence functionals computed for independent outliers

The functions plotted in figure 2 were obtained after standardizing the influence functionals of each estimator by a commom factor, and therefore they represent any coordinate of the IF $_{A O, 1}$. That is, we just compare the QMLE quadratically unbounded curve $\left(\zeta^{2}-1\right)$, and the CM-estimators bounded curve $(6 c / B)\left(\zeta^{2} / \lambda-2\left(\zeta^{2} / \lambda\right)^{2}+\right.$ $\left.\left(\zeta^{2} / \lambda\right)^{3}\right) I_{\left(\zeta^{2} \leq \lambda\right)}-1$. Note that both curves reflect the form of their corresponding $\tilde{\boldsymbol{\Psi}}$ functions.

We now address the problem of tuning the CM-estimators for ARCH models. The approach used here is the same previously used to tune the CM-estimators for other statistical models. The idea is to find a value for the tuning constant $\lambda$ which will balance bias robustness and efficiency robustness at a chosen core process. 
Given a $\rho$ function, our method for choosing a value for the tuning constant $c$ such that our estimators would have good local robustness properties, consist in choosing the constant $\lambda$ which would imply in a reasonable asymptotic relative efficiency at the Gaussian process and a small GES $_{A O, 1}$ based on the special case of contaminating measures $\left\{\delta_{\zeta}\right\}$. We note that it is sufficient to tune the CM-estimator of $\alpha_{0}$ since we may write the conditional variance as $\sigma_{1}^{2}=\alpha_{0}+\alpha_{1} x_{0}^{2}+\alpha_{2} x_{-1}^{2}+\ldots+\alpha_{p} x_{-p+1}^{2}=$ $\alpha_{0}\left(1+\phi_{1} x_{0}^{2}+\phi_{2} x_{-1}^{2}+\ldots+\phi_{p} x_{-p+1}^{2}\right)$, and, therefore, just the first entry of the $\operatorname{GES}_{A O, 1}$ is used in the tuning procedure. Figure 3 illustrates this idea. This figure shows the value of the relative asymptotic efficiency and the values for the gross error sensitivity plotted against the tuning constant $\lambda$. Note that as $\lambda \rightarrow \infty$ the efficiency $\rightarrow 1$.

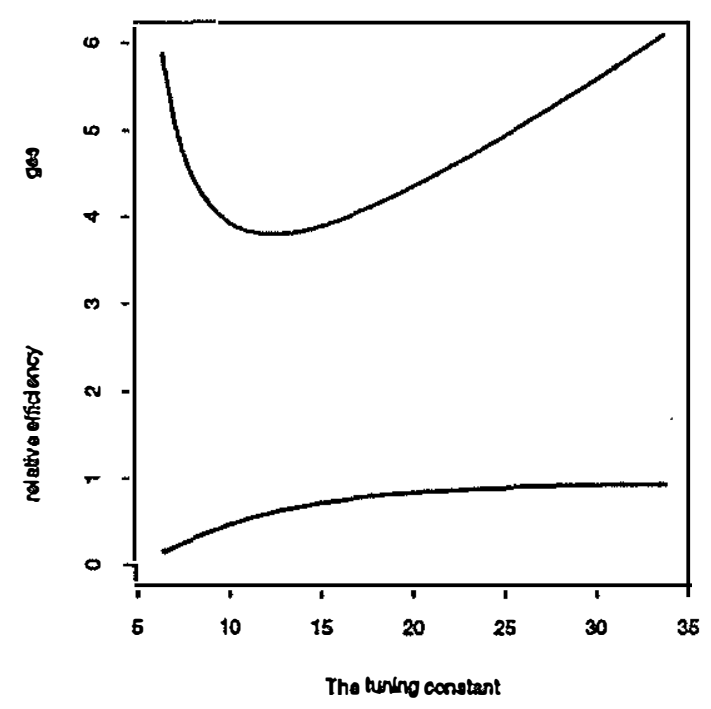

Figure 3

The CM-estimators relative efficiency and GES under isolated outliers plotted against the tuning constant $\lambda$ 
Table 1, besides giving a selection of values for $\lambda$, the corresponding constants $c$, and the efficiency, also gives the $\operatorname{GES}_{A O, 1}$. For the Biweighted $\rho$ function the choice of $\lambda=14.4$ implies the smaller gross error sensitivity, and the choice of $\lambda=26.2$ corresponds to $91 \%$ efficient estimates at the normal model.

\section{Bias under Contaminations}

Outliers are observations which may have not been generated by the true process that have generated the majority of the data (Huber, 1981, Hampel et al., 1986). As we have already stressed, even though GARCH-type models can handle very well data presenting volatility clusters, these (important) extreme observations will certainly distort the estimates and the forecasts provided by classical estimates. Such spurious observations may produce heavily biased estimates and, as illustrated by our simulations, the QMLE of ARCH models seem to be very sensitive to these atypical observations. The main message of this section is that whether or not we are able to explain the unusual data points, we do need robust procedures to resist to them.

We show here the results of a small Monte Carlo experiment carried out to investigate the bias robustness of the $91 \%$ efficient Biweighted CM-estimators. The data $y_{t}^{\gamma}, t=1,2, \ldots, n$, were generated according to a Gaussian ARCH(1) model contaminated with additive outliers:

$$
\begin{aligned}
& y_{t}^{\gamma}=\left(1-z_{t}^{\gamma}\right) x_{t}+z_{t}^{\gamma} \zeta, \\
& x_{t} \mid \mathrm{x}_{t-1}^{-1} \sim \mathrm{N}\left(0, \sigma_{t}^{2}\right), \\
& h_{t}=\alpha_{0}+\alpha_{1} x_{t-1}^{2}, \quad t=1,2, \ldots, n,
\end{aligned}
$$

as given in section 2 , and where $\zeta$ is the contaminating value. We use the notation $\mathrm{A} 1\left(\alpha_{0}, \alpha_{1}, \gamma, \zeta\right)$ to represent the model (27). The 
true parameters were set either equal to $\left(\alpha_{0}=1, \alpha_{1}=0.3\right)$, or equal to $\left(\alpha_{0}=1, \alpha_{1}=0.9\right)$. The proportion of contamination $\gamma$ was set equal to $0 \%$ and $3 \%$. The magnitudes of the additive outliers $\zeta$ were set equal to 12 and 32 , corresponding approximately to 10 times the unconditional standard deviation associated with models $\mathrm{A} 1(1.0,0.3,0, \cdot)$ and $\mathrm{A} 1(1.0,0.9,0, \cdot)$. For each model we generated 200 series of size $n=500$ representing each approximately two years of financial data. For each series we computed the classical and the robust estimates. All calculations were done using the S language and the S-Plus package (Statistical Sciences, 1995).

Table 2 reports the means and the standard deviations (inside parentheses) of the estimates. The results in the left side of table 2 correspond to the model $\mathrm{A} 1(1.0,0.3, \cdot, \cdot)$. The right side of table 2 contains the results related to the model $\mathrm{A} 1(1.0,0.9, \cdot, \cdot)$.

\section{Table 2}

Means and Standard Deviations of the QMLE and the CM-Estimators of a Gaussian ARCH(1) Process

Contaminated with Additive Outliers

\begin{tabular}{ccccc}
\hline & $\mathrm{A} 1(1.0,0.3,0,)$. & $\mathrm{A} 1(1.0,0.3,3,12)$ & $\mathrm{A} 1(1.0,0.9,0,)$. & $\mathrm{A} 1(1.0,0.9,3,32)$ \\
\hline QMLE $\alpha_{0}$ & 0.996970 & 5.527628 & 1.036407 & 34.543870 \\
& $(0.089958)$ & $(0.697446)$ & $(0.122920)$ & $(3.436384)$ \\
QMLE $\alpha_{1}$ & 0.302125 & 0.000000 & 0.859140 & 0.000000 \\
& $(0.053812)$ & $(0.000000)$ & $(0.091163)$ & $(0.000000)$ \\
\hline CM $\alpha_{0}$ & 0.998349 & 1.251337 & 1.039783 & 1.069756 \\
& $(0.092022)$ & $(0.135713)$ & $(0.135513)$ & $(0.147728)$ \\
CM $\alpha_{1}$ & 0.306290 & 0.028944 & 0.857866 & 0.685921 \\
& $(0.056658)$ & $(0.064532)$ & $(0.096756)$ & $(0.112217)$ \\
\hline
\end{tabular}

We first observe that the classical and the robust estimators gave basically the same results under no contamination for both models. 
However, under just the little proportion of $3 \%$ of contamination, the QMLE broke down while the CM-estimators remained fairly stable.

Under the model A1 $(1.0,0.3,3,12)$ the classical estimates of $\alpha_{0}$ increased and those of $\alpha_{1}$ decreased to zero. In fact, a small percentage (11\%) of the QMLE estimates of $\alpha_{1}$ went to the boundary 1 (mean 1.1 and standard error 0.6). The numbers given in table 2 for the QMLE of $\alpha_{1}$ are the averages over the set of results which went to zero. We believe that we should not mix the two behaviors. On the other hand, the robust estimates of $\alpha_{0}$ showed small bias while the estimates of $\alpha_{1}$ were still able to capture the dynamics of the process.

For the model $\mathrm{A} 1(1.0,0.9,3,32)$ the results were more dramatic. The classical estimates of $\alpha_{0}$ exploded and those of $\alpha_{1}$ again either went to zero $(94 \%)$ or to 1 (6\%, mean 1.3 and standard error 1.9$)$. Not surprisingly, the robust estimators provided very good estimates: the average CM-estimates of $\alpha_{0}$ and $\alpha_{1}$ were respectively 1.069756 and 0.685921 .

It is also of interest to investigate an estimator ability to estimate the unconditional variance of the process. Let $\sigma_{n}^{2}$ represent the estimator of the unconditional variance of an ARCH process. For example, in the case of a Gaussian ARCH(1) model and the CM-estimators, $\sigma_{n}^{2}$ is obtained by computing $\frac{\alpha_{0}}{1-\alpha_{1}}$ using the CMestimates. Table 3 shows the means and standard deviations of the classical and robust estimates of $\sigma_{n}^{2}$ of model A1 $(1.0,0.3, \cdot, \cdot)$. Note that under the nominal process, when the true unconditional variance is equal to 1.4286 , both estimators provided good answers. However, under the contaminated model just the CM-estimators were able to provide a good estimate. 
Beatriz V.M. Mendes and A.M. Duarte Júnior

Table 3

Means and Standard Deviations of the Unconditional Variance Estimates

\begin{tabular}{lccc}
\hline A1 $(1.0,0.3, . .)$. & \multicolumn{3}{c}{ A1 $(1.0,0.3,3,12)$} \\
\hline QMLE & CM & QMLE & CM \\
\hline 1.4333 & 1.4446 & 5.5276 & 1.2892 \\
$(0.1363)$ & $(0.1411)$ & $(0.6974)$ & $(0.1157)$
\end{tabular}

\section{Applications}

In this section we make use of four Brazilian financial series to show the usefulness of the robust procedure proposed and to compare its performance to the classical procedure for GARCH models. We focus on the accurate estimation and prediction of the conditional volatility of the series. In other words, we want to obtain reliable estimates for the parameters of the GARCH model, which can resist to extreme data points (as illustrated in figure 1) and can adequately express the dynamics existing in the data.

For each of the four series analyzed in this section, we consider the estimation of the model

$$
y_{t}=C+x_{t}, \quad t=1, \ldots, n,
$$

where $y_{t}$ are the observations and $x_{t}$ follows a GARCH$(1,1)$ model. We estimate the model using the QMLE with the standard normal and the t-student densities. The number of degrees of freedom of the t-student distribution is jointly estimated.

The four data sets used are daily returns of a stock or index. They were obtained by taking the percentages of the logarithm of the ratio of two consecutive closing prices. 


\section{Analysis of the C-bond}

The first data set contains observations of a Brazilian Brady bond, the C-bond, and consists in 1066 observations from April/1994 to July/1998. Figure 1 shows the series. We observe at least two extreme observations just after the Mexican crisis of 1994, and two other spikes at the end of 1997, both followed by periods of high volatility.

The estimated values for $\left(\alpha_{0}, \alpha_{1}, \beta_{1}\right)$ under the three estimation procedures (QMLE-Normal, QMLE-t-student, and Robust) are respectively $(0.000008808,0.1689,0.8149),(0.000002734,0.0513$, $0.8906)$, and $(0.000004683,0.0708,0.6748)$.

The robust estimates are not so affected by the extreme points. This fact can be better appreciated by examining the figure 4 , where we plot the anualized volatilities obtained from the three estimation procedures. We observe that the conditional volatility estimated by the robust method possess a lower level and seems to reflect better the effects of typical days. This fact occurs probably because the estimators downweight the effect of outliers which is exactly the behavior expected from an estimator which tries to reflect the dynamics contained in the majority of the data. This figure gives us a better idea of the effects of the different estimation procedures and give more information than just the examination of the estimated values of the parameters.

The goodness of fit test of Ljung -Box (Ljung e Box, 1978) can be used to test the null hypotheses that the squared standardized residuals (standardized using the estimated conditional volatility $\hat{\sigma}_{t}$ ) are free of volatility clusters. The test statistic should be compared to a percentil of a chi-squared random variable. The number of degrees of freedom depend upon the data and should be set a priori. Here we used 12 lags and for a test with $5 \%$ significance level the cor- 
responding critical value is 21.02 . These results are given in table 4 for the three fits.

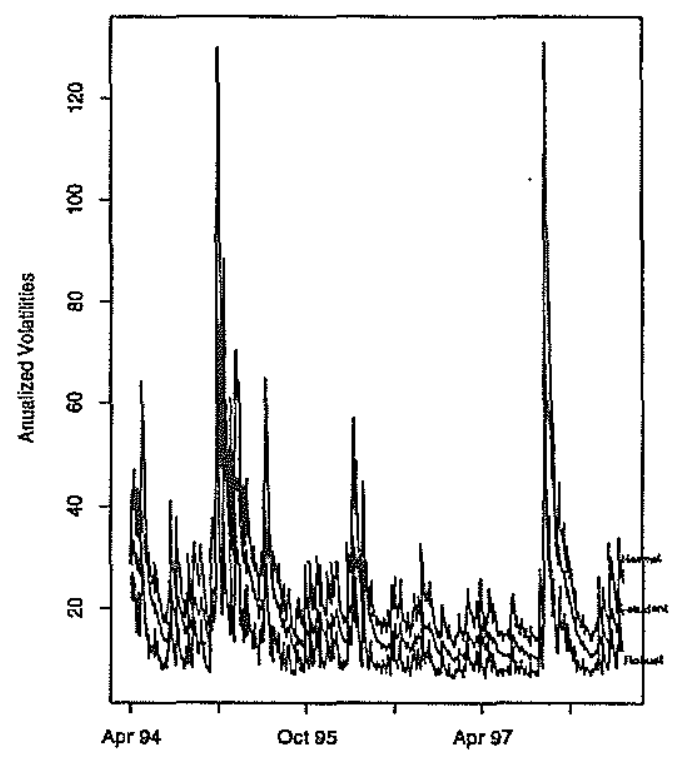

Figure 4

Anualized volatilities from the classical and robust fits

Table 4 gives the values of the kurtosis, the Ljung-Box statistics $\left(L B_{12}^{2}\right)$ and the corresponding $\mathrm{p}$-value for the squared original data and for the squared standardized residuals from the three fits. We observe that the null hypothesis is rejected for the normal and tstudent fits, but it is not rejected for the robust fit. This means that there is still some dynamics in the data which were not captured by the classical fits. This fact is also supported by the large kurtosis values found for these residuals. Finally, we note that the value of $L B_{12}^{2}$ for the squared original data is much higher before the fits, indicating the appropriateness of the GARCH model. 
Table 4

The Ljung-Box Test for the Three Fits of the C-bond

\begin{tabular}{ccc}
\hline \multicolumn{3}{c}{ Squared original data } \\
Kurtosis & $L B_{12}^{2}$ & p-value \\
13.93 & 265.89 & 0.0000 \\
\hline Squared standardized residuals (Normal) \\
Kurtosis & $L B_{12}^{2}$ & p-value \\
5.12 & 22.54 & 0.0319 \\
\hline Squared standardized residuals (t-student -4 & degrees of freedom) \\
Kurtosis & $L B_{12}^{2}$ & p-value \\
8.59 & 26.23 & 0.0100 \\
\hline Squared standardized residuals (Robust) \\
Kurtosis & $L B_{12}^{2}$ & p-value \\
3.38 & 16.54 & 0.1676 \\
\hline
\end{tabular}

Since we cannot observe the conditional volatility, it is not possible to check the reliability of the estimates provided by the three methods. However, we can compare some measures derived from the fact that the conditional expectation of $y_{t}^{2}$ is $C^{2}+\sigma_{t}^{2}$. Then, an estimate for the next squared observation may be computed using an estimate $\hat{\sigma_{t}^{2}}$ for $\sigma_{t}^{2}$, the estimated parameters and the observations up to day $t-1$. We can interpret the quantity $y_{t}^{2}-C^{2}-\sigma_{t}^{2}$ as a prediction error. If positive at time $t$, it means that the procedure underestimated the volatility at time $t$. If negative, it means that the volatility was overestimated.

In table 5 we give some descriptive statistics for the prediction error (times 100) for the three fits. 
Beatriz V.M. Mendes and A.M. Duarte Júnior

Table 5

Descriptive Statistics for the Predictions Errors

( $\times 100)$ Given by the Methods

Minimum 1st.Quartile Median Mean 3rd.Quartile Maximum

\begin{tabular}{llllll}
\hline \multicolumn{6}{c}{ Normal } \\
-0.67890 & -0.020350 & -0.009927 & -0.001814 & 0.000155 & 2.5250 \\
\hline \multirow{5}{*}{ T-student } \\
-0.22990 & -0.008739 & -0.003774 & 0.064100 & 0.008469 & 2.5490 \\
\hline \multicolumn{5}{c}{ Robust } \\
-0.23200 & -0.003000 & 0.001320 & 0.068800 & 0.015790 & 2.5510 \\
\hline
\end{tabular}

We note in table 5 that the classical prediction errors possess negative median and thus systematically overestimate the volatility. In fact, this behavior was expected since the classical estimators give the same weight to all observations, including the outliers. On the other hand, the statistics for the prediction error from the robust fit indicate a distribution much more concentrated around its median which is very close to zero.

We can also compute the more important quantity $\frac{1}{n} \sum\left(y_{t}^{2}-C^{2}-{\hat{\sigma_{t}^{2}}}^{2}\right)^{2}$, which should be small if the estimates are accurate. However, if there is an outlier at some time $t$, say $t=K$, the corresponding squared error $\left(y_{K}^{2}-C^{2}-\hat{\sigma}_{K}^{2}\right)^{2}$ may be very large. From the robust perspective, this is the correct thing to do. A better statistic may be either a trimmed (5\%) mean or the median of the absolute values of $\left(y_{t}^{2}-C^{2}-\hat{\sigma}_{t}^{2}\right)$. Table 6 shows these statistics for the three fits. 
Robust Estimation for ARCH Models

Table 6

The Squared Distances for the Three Estimation Procedures $(\times 10000)$

\begin{tabular}{lccc}
\hline & $\begin{array}{c}\text { Mean Squared } \\
\text { Error }\end{array}$ & $\begin{array}{c}\text { \%5 Trimmed Mean } \\
\text { Squared Error }\end{array}$ & $\begin{array}{c}\text { Median of } \\
\text { Abs. Error }\end{array}$ \\
\hline Normal & 0.0193 & 0.00127 & 0.01500 \\
T-student & 0.0197 & 0.00077 & 0.00880 \\
Robust & 0.0202 & 0.00072 & 0.00072 \\
\hline
\end{tabular}

We observe that using the two robust statistics the robust procedure stands out as the method producing the smaller long run error for the majority (here $90 \%$ ) of the data. This means that the robust procedure gets the right information given by most of the data. We would like to observe that we do not consider as a measure of goodness of fit the number of observations falling outside of an interval defined by \pm 2 conditional standard deviations for this measure is also misleading by the same reasoning just given.

\section{Analysis of the Cocoa}

The results obtained when estimating the volatility of the other three data sets were very similar to those obtained for the C-bond. Typically, the classical estimates provided inflated volatilities caused by the presence of atypical points, while the robust volatility presented always a smaller level. Thus, for the other series we give just the more important results.

The second data set contains daily returns of Cocoa, and consists in 2117 observations.

The estimated values for $\left(\alpha_{0}, \alpha_{1}, \beta_{1}\right)$ under the three estimation procedures (QMLE-Normal, QMLE-t-student, and Robust) are respectively $(0.00000159,0.030771,0.964242),(0.00000122,0.018760$, $0.954500)$, and $(0.00000432,0.036584,0.596881)$. 
The plot the anualized volatilities obtained from the three estimation procedures presented the same behavior shown by the fit of the first data. The conditional volatility estimated by the robust method showed a lower level, followed by the t-student and then the normal, reflecting better the effects of typical days.

The goodness of fit test of Ljung -Box (Ljung e Box, 1978) are given in table 7 for the three fits. We observe that the null hypothesis is rejected for the normal and t-student fits, but it is not rejected for the robust fit.

\section{Table 7}

The Ljung-Box Test for the Three Fits of the C-bond

\begin{tabular}{ccc}
\hline \multicolumn{3}{c}{ Squared original data } \\
Kurtosis & $L B_{12}^{2}$ & $\mathrm{p}$-value \\
6.06 & 95.34 & 0.0000 \\
\hline Squared standardized residuals (Normal) \\
Kurtosis & $L B_{12}^{2}$ & $\mathrm{p}$-value \\
4.33 & 9.47 & 0.6622 \\
\hline Squared standardized residuals (t-student -4 & degrees of freedom) \\
Kurtosis & $L B_{12}^{2}$ & p-value \\
4.36 & 9.38 & 0.6700 \\
\hline Squared standardized residuals (Robust) & p-value \\
Kurtosis & $L B_{12}^{2}$ & 0.5095 \\
9.07 & 11.22 &
\end{tabular}

In table 8 we give some the statistics based on the the prediction error for the three fits. 
Robust Estimation for ARCH Models

Table 8

The Squared Distances for the Three Estimation Procedures $(\times 10000)$

\begin{tabular}{lccc}
\hline & $\begin{array}{c}\text { Mean Squared } \\
\text { Error }\end{array}$ & $\begin{array}{c}\text { \%5 Trimmed Mean } \\
\text { Squared Error }\end{array}$ & $\begin{array}{c}\text { Median of } \\
\text { Abs. Error }\end{array}$ \\
\hline Normal & 0.0061 & 0.0008 & 0.000186 \\
T-student & 0.0063 & 0.0005 & 0.000100 \\
Robust & 0.0068 & 0.0006 & 0.000051 \\
\hline
\end{tabular}

We observe that the $5 \%$ trimmed mean squared error chose the t-student QMLE as the best fit, whereas the median of absolute erros chose the robust procedure.

\section{Analysis of Telebras-PN}

The second data set are returns of the stock Telebras-PN which contains 988 observations.

The estimated values for $\left(\alpha_{0}, \alpha_{1}, \beta_{1}\right)$ under the three estimation procedures (QMLE-Normal, QMLE-t-student, and Robust) are respectively (00003463, 0.174199, 0.795329), (0.00002191, 0.137564, $0.806156)$, and $(0.0,0.0708,0.6748)$.

The plot the anualized volatilities obtained from the three estimation procedures presented the same behavior previously observed. For this data set the two classical volatilities were very close, whereas the robust one lied at a smaller level, well separated from the others.

The goodness of fit test of Ljung -Box (Ljung e Box, 1978) are not given, but we report that the null hypothesis is not rejected for all three fits with p-values of 0.1853 (normal), 0.1689 (t-student), and 0.2074 (robust). The statistics involving the prediction error are given in table 9 . 
Beatriz V.M. Mendes and A.M. Duarte Júnior

Table 9

The Squared Distances for the

Three Estimation Procedures $(\times 10000)$

\begin{tabular}{lccc}
\hline & $\begin{array}{c}\text { Mean Squared } \\
\text { Error }\end{array}$ & $\begin{array}{c}\text { \%5 Trimmed Mean } \\
\text { Squared Error }\end{array}$ & $\begin{array}{c}\text { Median of } \\
\text { Abs. Error }\end{array}$ \\
\hline Normal & 0.1582 & 0.0096 & 5.02 \\
T-student & 0.1563 & 0.0083 & 4.36 \\
Robust & 0.1612 & 0.0074 & 2.67 \\
\hline
\end{tabular}

We observe that the two robust statistics chose the robust CMestimators as the method producing the smaller long run error for the vast majority of the trading days.

\section{Analysis of the US Dollar/Real exchange rate}

The fourth data set contains observations of the US dollars to Brazilian Real exchange rate, and consists in 996 observations.

The estimated values for $\left(\alpha_{0}, \alpha_{1}, \beta_{1}\right)$ under the three estimation procedures (QMLE-Normal, QMLE-t-student, and Robust) are respectively $(0.0000000067,0.13,0.87),(0.0000000049,0.06,0.84)$, and $(0.0000000000011,0.138,0.805)$.

The plot the anualized volatilities obtained from the three estimation procedures were closer for this data. The conditional volatility estimated by the t-student method lied in between the other two.

In table 10 we give some the statistics based on the the prediction error for the three fits. There is not much difference between the tstudent and the robust fit. In fact, the number of degrees of freedom estimated for the t-student was just 2 . 
Robust Estimation for ARCH Models

Table 10

The Squared Distances for the

Three Estimation Procedures $(\times 10000)$

\begin{tabular}{lccc}
\hline & $\begin{array}{c}\text { Mean Squared } \\
\text { Error }\end{array}$ & $\begin{array}{c}\text { \%5 Trimmed Mean } \\
\text { Squared Error }\end{array}$ & $\begin{array}{c}\text { Median of } \\
\text { Abs. Error }\end{array}$ \\
\hline Normal & 0.0002 & 0.0000 & 0.001 \\
T-student & 0.0002 & 0.0000 & 0.000 \\
Robust & 0.0002 & 0.0000 & 0.000 \\
\hline
\end{tabular}

\section{Conclusions}

Financial data are fat tailed, possess volatility clusters, and present occasional extreme observations. Even though GARCH models provide a very good description of the underlying process generating the data, the Gaussian maximum likelihood estimates become distorted and show large variability under misspecifications. We proposed a new robust and efficient estimator which does not assume a particular likelihood function to maximize and no additional parameters to estimate. On the contrary, the user is free to choose a loss function better suited for the data at hand. In this sense it has no chance to be misspecified.

We obtained their robustness properties and showed how to tune the estimates to obtain high efficiency at the core process. Estimates for the standard errors of the estimates can be constructed using the asymptotic variance. We showed using simulations that the robust estimates perform very well under no contaminations and that the gains in bias under small percentages of contamination of moderate size are considerable.

Four sets of real data were used to compare the classical and the robust estimates when estimating and forecasting the volatility of 
the series. Typically the estimated conditional volatilities, classical and robust, differ in level and in the identification of the dynamics of the series. The robust procedure provided estimates not inflated by the presence of a few outliers.

Likewise all robust estimates their computations are more time consuming, and more research on algorithms to compute the estimates are needed. We plan further research on extending the concept of the CM-estimation to generalizations of GARCH models.

\section{Acknowledgments}

The first author acknowledges partial financial support from FAPERJ and CNPq/PRONEX.

Submitted on March 1999 and revised on May 1999.

\section{References}

Badrinath, S.G. \& Chattergee, S. 1988. "On measuring skewness and elongation in common Stock return distributions: the case of the market index". Journal of Business, 61:451-472.

Basawa, I.V.; Feigin, P.D. \& Heyde, C.C. 1976. "Asymptotic properties of maximum likelihood estimators for stochastic processes". Sankhya, Series A, 38:259-270.

Bollerslev, T. 1986. "Generalized autoregressive conditional heteroskedasticity". Journal of Econometrics, 31:307-327.

. 1987. "A conditionally heteroskedastic time series model for speculative prices and rates of return". The Review of Economics and Statistics, 69:542-547.

\& Wooldridge, J.M. 1992. "Quasi-maximum likelihood estimation and inference in dynamic models with time-varying covariances". Econometric Reviews, 11:143-172. 
Deb, P. 1996. "Finite sample properties of maximum likelihood and quasi-maximum likelihood estimators of EGARCH models". Econometric Reviews, 15(1):51-68.

Donoho, D.L. \& Huber, P.J. 1983. "The notion of breakdown point". In: Bickel, P.J.; Doksum, K.A. \& Hodges Jr, J.L. (eds.). Festschrift for Erich L. Lehmann. Wadsworth: Belmont, California, $157-184$.

Engle, R.F. 1982. "Autoregressive conditional heterocedasticity with estimates of the variance of United Kingdom inflation". Econometrica, 50(4):987-1007.

; Lilien, D.M. \& Robins, R.P. 1987. "Estimating time varying risk premia in the term structure: the ARCH-M model". Econometrica, 55:391-407.

\& González-Rivera, G. 1991. "Semi-parametric estimation of ARCH model". Journal of Business and Economic Statistics, 9(4):345-358.

Gallant, A.R.; Hsieh, D. \& Tauchen, G. 1989. "On fitting a recalcitrant series: the pound/dollar exchange rate". Unpublished manuscript.

Hampel, F.R. 1974. "The influence curve and its role in robust estimation". Journal of the American Statistical Association, 69:383-393.

; Ronchetti, E.M.; Rousseeuw, P.J. \& Stahel, W.A. 1986. Robust Statistics: The Approach Based On Influence Functions, Wiley, New York.

Huber, P.J. 1981. "Robust Statistics". Wiley, New York.

Kent, J.T. \& Tyler, D.E. 1991. "Redescending mestimators of multivariate location and scatter". Annals of Statistics, 19(4):21022119 . 
\& Tyler, D.E. 1996. "Constrained mestimators for multivariate location and scatter". Annals of Statistics, 24(3):13461370.

Koenker, R. \& Zhao, Q. 1996. "Conditional quantile estimation and inference for ARCH models". Econometric Theory, 12:793-813.

Lee, S.W. \& Hansen, B.E. 1994. "Asymptotic theory for the GARCH $(1,1)$ quasi-maximum likelihood estimator". Econometric Theory, 10:29-52.

Linton, O. 1997. "An asymptotic expansion in the $\operatorname{GARCH}(1,1)$ model". Econometric Theory, 13:558-581.

Ljung, G.M. \& Box, G.E.P. 1978. "On a measure of lack of fit in time series models". Biometrika, 65(2):297-303.

Lumsdaine, R.L. 1995. "Finite sample properties of the maximum likelihood estimator in $\operatorname{GARCH}(1,1)$ and IGARCH(1,1) models: a Monte Carlo investigation". Journal of Business and Economic Statistics, 13:1-10.

Martin, R.D. \& Yohai, V.J. 1986. "Influence functionals for time series". Annals of Statistics, 14(3):781-818.

Mendes, B.V.M. 1995. "Constrained M-estimation for linear regression models". PhD. Thesis. Rutgers University, New Jersey, USA.

1996. "Robustness aspects of ARCH models estimates". Proceedings of the XIV Latin American Meeting of the Econometric Society, IMPA, Rio de Janeiro.

\& Tyler, D.E. 1996. "Constrained M-estimation for regression". In: Helmut Rieder ed. Robust Statistics, Data Analysis, and Computer Intensive Methods. Lecture Notes in Statistics, Springer Verlag, 109:299-320. 
Nelson, D.B. 1991. "Conditional heteroscedasticity in asset returns". Econometrica, 59:347-370.

\& Foster, D.P. 1994. "Asymptotic filtering theory for univariate ARCH models". Econometrica, 62(1):1-41.

MathSoft. 1996. "S+GARCH user's manual". Data Analysis Products Division, MathSoft, Seattle.

Rabemananjara, R. \& Zakoian, J.M. 1993. "Threshold ARCH models and asymmetries in volatility". Journal of Applied Econometrics, 8:31-49.

Schewert, G.W. 1989. "Why does Stock market volatility change over time?" Journal of Finance, 44:1115-1154.

Statistical Sciences, Inc. 1995. "S-Plus user's manual". Version 3.3 for Windows. Statistical Sciences, Inc., Seattle, WA, USA.

Weiss, A.A. 1986. "Asymptotic theory for ARCH models: estimation and testing. Econometric Theory, 2:107-131.

Zakoian, J.M. 1991. "Threshold heteroskedastic models". D.P. INSEE.

\section{APPENDIX}

Proof of Theorem 3.1.

A. Under strict inequality constrain

Using the $\tilde{\boldsymbol{\Psi}}$ functions (14) and (15) we obtain

$$
\frac{\partial}{\partial \mathbf{t}}\left[\tilde{\boldsymbol{\Psi}}\left(\mathbf{x}_{1}, \mathbf{t}\right)\right]=b(\tilde{\boldsymbol{\Psi}}) \frac{\mathbf{z}_{1}}{\sigma_{1}^{2}} \frac{\mathbf{z}_{1}^{\prime}}{\sigma_{1}^{2}}
$$

where $\mathbf{z}_{1}^{\prime}=\left(1, x_{0}^{2}, x_{-1}^{2}, \ldots, x_{-q+1}^{2}\right)$, and $b(\tilde{\Psi})=(-2) u^{\prime}\left(x_{1}^{2} / \sigma_{1}^{2}\right)$ $\left(x_{1}^{2} / \sigma_{1}^{2}\right)^{2}-4 u\left(x_{1}^{2} / \sigma_{1}^{2}\right)\left(x_{1}^{2} / \sigma_{1}^{2}\right)+1$. 
Let $B\left(\tilde{\Psi} ; \mathbf{t}_{0}\right)$ represent the unconditional expectation $\mathrm{E}[-b(\tilde{\Psi})]=-\mathrm{E}\left[\mathrm{E}\left[b(\tilde{\Psi}) \mid \mathcal{I}_{0}\right]\right.$ at $\mathbf{t}_{0}$, where $\left(\mathcal{I}_{0}\right)$ represents all past information. Since $\mathbf{C}=\mathrm{E}\left[\partial / \partial \mathrm{t} \tilde{\Psi}\left(\mathrm{x}_{1}, \mathrm{t}\right)\right]_{\mathbf{t}=\mathbf{t}_{0}}$, the result follows from (17).

B. Under equality constrain.

Let $A=\left[\int 2 u\left(x_{1}^{2} / \sigma_{1}^{2}\right)\left(x_{1}^{2} / \sigma_{1}^{2}\right)\left(1 / \sigma_{1}^{2}\right) d \mu\left(\mathrm{x}_{1}\right)\right] /\left[\int 1 / \sigma_{1}^{2} d \mu\left(\mathrm{x}_{1}\right)\right]$. Using the $\tilde{\boldsymbol{\Psi}}$ functions (refeq16) and (refeq18) we obtain

$$
\frac{\partial}{\partial \mathbf{t}}\left[\tilde{\Psi}\left(\mathbf{x}_{1}, \mathbf{t}\right)\right]=\mathbf{b}(\tilde{\Psi}) \frac{\mathbf{z}_{1}}{\sigma_{1}^{2}} \frac{\mathbf{z}_{1}^{\prime}}{\sigma_{1}^{2}}
$$

where $\mathbf{b}$ is a diagonal matrix with diagonal $\left(-u\left(x_{1}^{2} / \sigma_{1}^{2}\right)\left(x_{1}^{2} / \sigma_{1}^{2}\right) \sigma_{1}^{2}\right.$, $\left.b^{*}, b^{*}, \ldots, b^{*}\right)$, where $b^{*}=-2 u^{\prime}\left(x_{1}^{2} / \sigma_{1}^{2}\right)\left(x_{1}^{2} / \sigma_{1}^{2}\right)^{2}-4 u\left(x_{1}^{2} / \sigma_{1}^{2}\right)\left(x_{1}^{2} / \sigma_{1}^{2}\right)+$ A.

Let $\mathrm{B}\left(\tilde{\boldsymbol{\Psi}} ; \mathbf{t}_{0}\right)$ represent the unconditional expectation $\mathrm{E}[-\mathbf{b}(\tilde{\Psi})]=-\mathrm{E}\left[\mathrm{E}\left[\mathbf{b}(\tilde{\Psi}) \mid \mathcal{I}_{0}\right]\right.$ at $\mathbf{t}_{0}$, which is a diagonal matrix with diagonal $\left(\mathrm{E} u\left(x_{1}^{2} / \sigma_{1}^{2}\right)\left(x_{1}^{2} / \sigma_{1}^{2}\right) \sigma_{1}^{2}, B^{*}, B^{*}, \ldots, B^{*}\right)$, and where $B^{*}=\mathrm{E}\left(-b^{*}\right)$. Part 2 then follows from (refeqich).

Proof of Theorem 3.2. From Martin and Yohai (1986), the asymptotic variance-covariance matrix of a $\tilde{\boldsymbol{\Psi}}$ estimate is given by

$$
\mathrm{V}_{0}+\sum_{l=1}^{\infty}\left(\mathrm{V}_{l}+\mathrm{V}_{l}^{T}\right)
$$

where

$$
\mathrm{V}_{l}=\operatorname{var}\left[\mathbf{I C H}\left(\mathbf{y}_{1}\right), \mathbf{I C H}\left(\mathbf{y}_{1+l}\right)\right] .
$$

Since under the true process, $\mathrm{E}\left[\mathbf{I C H}\left(\mathrm{y}_{l}\right)\right]=0$, and $\operatorname{var}\left(\mathbf{I C H}\left(\mathbf{y}_{1}\right)\right.$, $\left.\operatorname{ICH}\left(\mathrm{y}_{1+l}\right)\right)=0, \forall l \geq 1$, at both the strict inequality and the equality cases, the asymptotic variance-covariance matrix is just

$$
\mathrm{V}_{0}=\operatorname{var}\left(\mathbf{I C H}\left(\mathbf{x}_{1}\right)\right)=\mathrm{E}\left[\left(\mathbf{I C H}\left(\mathbf{x}_{1}\right)\right)^{2}\right] .
$$

Revista de Econometria 19 (1) Maio 1999 
Thus, using the results obtained in proof of Theorem 3.1 we have A. Under strict inequality constrain:

$\mathrm{E}\left(\mathbf{I C H}\left(\mathrm{x}_{1}\right)\right)^{2}=\frac{1}{B^{2}\left(\tilde{\boldsymbol{\Psi}} ; \mathbf{t}_{0}\right)}\left[\mathrm{E}\left(\frac{\mathbf{z}_{1}}{\sigma_{1}^{2}} \frac{\mathbf{z}_{1}^{\prime}}{\sigma_{1}^{2}}\right)\right]^{-1} \mathrm{E}\left(2 u\left(\frac{x_{1}^{2}}{\sigma_{1}^{2}}\right)\left(\frac{x_{1}^{2}}{\sigma_{1}^{2}}\right)-1\right)^{2}$

B. Under equality constrain:

$$
\mathrm{E}\left(\mathbf{I C H}\left(\mathrm{x}_{1}\right)\right)^{2}=\mathbf{B}\left(\tilde{\boldsymbol{\Psi}} ; \mathrm{t}_{0}\right)^{-2} \mathrm{E}[\mathbf{Z}]\left[\mathrm{E}\left(\frac{\mathbf{z}_{1}}{\sigma_{1}^{2}} \frac{\mathbf{z}_{1}^{\prime}}{\sigma_{1}^{2}}\right)\right]^{-1},
$$

where $\mathbf{Z}=\mathbf{v}^{\prime} \mathbf{v}$ is a $(p+1) \times(p+1)$ symmetric matrix, with $\mathbf{v}^{\prime}=\left(\left(\rho\left(x_{1}^{2} / \sigma_{1}^{2}\right)-\epsilon \rho(\infty)\right) \sigma_{1}^{2}\left(1 / \sigma_{1}^{2}\right), \quad\left(2 u\left(x_{1}^{2} / \sigma_{1}^{2}\right)\left(x_{1}^{2} / \sigma_{1}^{2}\right)-\right.\right.$ A) $\left.\left(x_{0}^{2} / \sigma_{1}^{2}\right), \ldots,\left(2 u\left(x_{1}^{2} / \sigma_{1}^{2}\right)\left(x_{1}^{2} / \sigma_{1}^{2}\right)-A\right)\left(x_{-p+1}^{2} / \sigma_{1}^{2}\right)\right)$.

Proof of Theorem 3.3. It may be shown that the sufficient conditions given in Theorem 4.2 of Martin and Yohai (1986) for the following expression

$$
\mathbf{I F}\left(\mu_{v}, \mathbf{T},\left\{\mu_{y}^{\gamma}\right\}\right)=\frac{-1}{k} \mathbf{C}^{-1} \sum_{j=0}^{\infty} \mathbf{G}_{j}^{k}
$$

hold for the CM-influence functionals for ARCH models.

A. Strict Inequality case: Here $-\mathbf{C}^{-1}=\left[M(\boldsymbol{\alpha})^{-1}\right] /\left[B\left(\tilde{\Psi} ; \mathrm{t}_{0}\right)\right]$ (see proof of Theorem 3.1), and the $\mathbf{G}_{j}^{k}$ 's are such that

$$
\mathbf{G}_{j}^{k}=\left\{\begin{array}{l}
\mathrm{E} \tilde{\boldsymbol{\Psi}}\left(\mathrm{v}_{1}^{1-j}, \mathbf{x}_{-j}, \mathbf{t}_{0}\right) \quad \text { if } 0 \leq j \leq k-1 \\
\mathrm{E} \tilde{\Psi}\left(\mathbf{x}_{1}^{1-j+k}, \mathbf{v}_{-j+k}^{1-j}, \mathbf{x}_{-j}, \mathbf{t}_{0}\right) \quad \text { if } j \geq k,
\end{array}\right.
$$


where the notation $x_{i}^{j}=\left(x_{i}, x_{i-1}, \ldots, x_{j}\right), j \leq i$. For independent outliers $(k=1)$ we have

$$
\mathbf{G}_{j}^{1}=\left\{\begin{array}{l}
\mathrm{E} \tilde{\boldsymbol{\Psi}}\left(\mathrm{v}_{1}^{1}, \mathrm{x}_{0}, \mathrm{t}_{0}\right) \quad \text { when } j=0 \\
\mathrm{E} \tilde{\boldsymbol{\Psi}}\left(\mathrm{x}_{1}^{2-j}, \mathrm{v}_{1-j}^{1-j}, \mathrm{x}_{-j}, \mathrm{t}_{0}\right) \quad \text { if for } j \geq 1
\end{array}\right.
$$

Thus

$$
\mathbf{G}_{0}^{1}=\mathrm{E}\left[\left(2 u\left(\frac{v_{1}^{2}}{\sigma_{1}^{2}}\right)\left(\frac{v_{1}^{2}}{\sigma_{1}^{2}}\right)-1\right) \frac{\mathbf{z}_{1}}{\sigma_{1}^{2}}\right],
$$

where the expectation is with respect to the measure $\mu_{x v}=\mu_{x} \mu_{v}$. All other vectors $\mathbf{G}_{j}^{1}$, are equal to $\mathbf{0}$. For example, $\mathbf{G}_{1}^{1}=$ $\mathrm{E} \tilde{\Psi}\left(\mathrm{x}_{1}^{1}, \mathrm{v}_{0}^{0}, \mathrm{x}_{-1}, \mathrm{t}_{0}\right)$ is equal to

$\mathrm{E} \tilde{\boldsymbol{\Psi}}\left(x_{1}, v_{0}, \mathbf{x}_{-1}, \mathbf{t}_{0}\right)=\mathrm{E}\left(2 u\left(\frac{x_{1}^{2}}{\sigma_{1}^{2}}\right)\left(\frac{x_{1}^{2}}{\sigma_{1}^{2}}\right)-1\right)\left[\begin{array}{l}1 \\ v_{0}^{2} \\ x_{-1}^{2} \\ \vdots \\ x_{-q+1}^{2}\end{array}\right]=\mathbf{0}$, $\mathbf{G}_{2}^{1}=\mathrm{E} \tilde{\Psi}\left(\mathrm{x}_{1}^{0}, \mathrm{v}_{-1}^{-1}, \mathbf{x}_{-2}, \mathrm{t}_{0}\right)=\mathbf{0}$, etc.

Thus, for independent outliers, $k=1$, the IF is just

$$
\mathbf{I F}_{A O, 1}\left(\mu_{v}, \mathbf{T}, \boldsymbol{\alpha}\right)=\frac{1}{B\left(\tilde{\Psi} ; \mathbf{t}_{0}\right)}[M(\alpha)]^{-1} \mathbf{G}_{0}^{1}
$$

where $M(\boldsymbol{\alpha})=\mathrm{E}\left[\frac{z_{1}}{\sigma_{1}^{2}} \frac{z_{1}^{\prime}}{\sigma_{1}^{2}}\right]$, and $B\left(\tilde{\Psi} ; \mathrm{t}_{0}\right)$ is as previously defined.

Let $\mathbf{z}_{1}^{(j)}=\left(1, v_{0}^{2}, v_{-1}^{2}, \ldots, v_{1-j}^{2}, x_{-j}^{2}, \ldots, x_{-p+1}^{2}\right)^{\prime}$ for $j<p$, where $\mathbf{z}_{1}^{(0)}=\mathbf{z}_{1}$. For $j \geq p, \mathbf{z}_{1}^{(j)}=\left(1, v_{0}^{2}, v_{-1}^{2}, \ldots, v_{-p+1}^{2}\right)^{\prime}$. For patches of $k$ 
outliers, $k \geq 2$, we have, for $0 \leq j \leq k-1, \mathrm{G}_{j}^{k}=\mathrm{E} \tilde{\Psi}\left(\mathrm{v}_{1}^{1-j}, \mathrm{x}_{-j}, \mathrm{t}_{0}\right)$. Then

$$
\begin{gathered}
\mathbf{G}_{0}^{k}=\mathrm{E} \tilde{\boldsymbol{\Psi}}\left(v_{1}, \mathbf{x}_{0}, \mathbf{t}_{0}\right)=\mathrm{E}\left[\left(2 u\left(\frac{v_{1}^{2}}{\sigma_{1}^{2}}\right)\left(\frac{v_{1}^{2}}{\sigma_{1}^{2}}\right)-1\right)\left(\frac{\mathbf{z}_{1}}{\sigma_{1}^{2}}\right)\right] \\
\left.\mathbf{G}_{1}^{k}=\mathrm{E} \tilde{\boldsymbol{\Psi}}\left(\mathbf{v}_{1}^{0}, \mathbf{x}_{-1}, \mathbf{t}_{0}\right)=\mathrm{E}\left(2 u\left(\frac{v_{1}^{2}}{\sigma_{1}^{2}}\right)\left(\frac{v_{1}^{2}}{\sigma_{1}^{2}}\right)-1\right) \mid \begin{array}{l}
1 \\
v_{0}^{2} \\
x_{-1}^{2} \\
\vdots \\
x_{-p+1}^{2}
\end{array}\right]\left(\frac{1}{\sigma_{1}^{2}}\right), \\
\mathbf{G}_{1}^{k}=\mathrm{E}\left[\left(2 u\left(\frac{v_{1}^{2}}{\sigma_{1}^{2}}\right)\left(\frac{v_{1}^{2}}{\sigma_{1}^{2}}\right)-1\right)\left(\frac{\mathbf{z}_{1}^{(1)}}{\sigma_{1}^{2}}\right)\right],
\end{gathered}
$$

etc. Thus

$$
\mathbf{G}_{j}^{k}=\mathrm{E}\left[\left(2 u\left(\frac{v_{1}^{2}}{\sigma_{1}^{2}}\right)\left(\frac{v_{1}^{2}}{\sigma_{1}^{2}}\right)-1\right)\left(\frac{\mathbf{z}_{1}^{(j)}}{\sigma_{1}^{2}}\right)\right],
$$

for $0 \leq j \leq k-1$. For $j \geq k$,

$$
\mathrm{G}_{j}^{k}=\mathrm{E} \tilde{\Psi}\left(\mathrm{x}_{1}^{1-j+k}, \mathrm{v}_{-j+k}^{1-j}, \mathbf{x}_{-j}, \mathrm{t}_{0}\right) .
$$

For example, for $j=k$

$$
\mathbf{G}_{k}^{k}=\mathrm{E} \tilde{\boldsymbol{\Psi}}\left(x_{1}, \mathbf{v}_{0}^{1-k}, \mathbf{x}_{-k}, \mathbf{t}_{0}\right)=\mathrm{E}\left[\left(2 u\left(\frac{x_{1}^{2}}{\sigma_{1}^{2}}\right)\left(\frac{x_{1}^{2}}{\sigma_{1}^{2}}\right)-1\right)\left(\frac{\mathbf{z}_{1}^{(1)}}{\sigma_{1}^{2}}\right)\right],
$$

which is zero by independence between $\mu_{x}$ and $\mu_{v}$ and since the expectation $\mathrm{E}\left[2 u\left(\frac{x_{1}^{2}}{\sigma_{1}^{2}}\right)\left(\frac{x_{1}^{2}}{\sigma_{1}^{2}}\right)-1\right]$ is equal to zero. Then, for all $j \geq k$, $\mathrm{G}_{j}^{k}$ are all equal to zero. Summarizing, for patches of outliers, $k \geq 2$, the influence functionals are equal to $\frac{1}{B}[M(\alpha)]^{-1} \sum_{j=0}^{k-1} G_{j}^{k}$, and the result follows. 